\title{
Relationships between rocky-reef fish assemblages, the sea urchin Diadema antillarum and macroalgae throughout the Canarian Archipelago
}

\author{
Fernando Tuya ${ }^{1, *}$, Arturo Boyra ${ }^{1}$, Pablo Sanchez-Jerez ${ }^{2}$, Carmen Barbera ${ }^{1}$, \\ Ricardo J. Haroun ${ }^{1}$ \\ ${ }^{1}$ Department of Biology, Faculty of Marine Sciences, Campus Tafira, University of Las Palmas de Gran Canaria, \\ 35017 Las Palmas, Canary Islands, Spain \\ ${ }^{2}$ Marine Biology Laboratory, University of Alicante, POB 99, 03080 Alicante, Spain
}

\begin{abstract}
In situ visual surveys using a hierarchical sampling design were carried out at 36 sublittoral rocky locations along the central-east Atlantic Canarian Archipelago to find relationships among (1) benthic primary producers, (2) the demographic structure of the herbivorous sea urchin Diadema antillarum Phillipi and (3) the trophic structure of coastal fish communities. Our correlation approach displayed a relationship between the lack of large macroinvertebrate-eating predatory fish and the increase in density of sea urchins, in addition to a decrease in fish richness. In contrast, increases in fast-growing plankton-feeding fish species were detected. The size structure of $D$. antillarum is dominated by small-to-intermediate sized sea urchins in environments with a high density of individuals, whereas low sea urchin density locations are characterized by the dominance of large sized individuals. The physical complexity of the substrate seems to play an important role in determining the local patchiness of $D$. antillarum. Finally, a non-linear decrease in the percentage of fleshy macroalgal cover with increasing density of $D$. antillarum was observed. We therefore propose $D$. antillarum as a key herbivorous species, which plays an important role in determining the structure of shallow, hard-substratum, infralittoral benthic communities throughout the Canary Islands.
\end{abstract}

KEY WORDS: Urchin-fish interactions · Trophic cascades · Diadema antillarum · Sea urchins · Fish assemblages $\cdot$ Macroalgae $\cdot$ Canary Islands

\section{INTRODUCTION}

Large, shallow, rocky areas, previously covered by fleshy erect algae, are transformed by sea urchins into overgrazed substrates dominated by encrusting coralline algae and algal turfs. These so-called urchingrazed barrens are often termed bare substrates (e.g. Mann 1982, McClanahan \& Sala 1997, Kingsford \& Battershill 1998, Sala et al. 1998, Shears \& Babcock 2003). These environments have been widely described in coastal temperate reefs (Vukovic 1982, Verlaque 1987, Andrew \& Underwood 1989, Francour 1994, Sala \& Zabala 1996, Sala et al. 1998, Babcock et al. 1999, Pinnegar et al. 2000, Shears \& Babcock 2003), as well as in tropical coral reefs (e.g. Hay 1984 McClanahan \& Kurtis 1991, Jackson 2001). These authors explain this as a direct consequence of an increase in inshore fishing pressure, since removal of target top predators (e.g. carnivorous fishes) can result in top-down trophic cascades and indirect effects on coastal marine assemblages (Sala et al. 1998, Pinnegar et al. 2000, Shears \& Babcock 2003). These studies mainly compare hard-substratum systems within longestablished Marine Protected Areas (MPAs) with non-protected zones subjected to artisanal fisheries (Pinnegar et al. 2000, Shears \& Babcock 2003).

Disturbances to the inshore areas of the Canarian Archipelago have increased due to the expanding 
tourist industry and the increased demand of fishery resources (Bortone et al. 1991, Falcón et al. 1996). However, no empirical evidence of the effect of these disturbances is available, as there are no data on the nearshore fish populations during the predevelopment era of the 1960s (Falcón et al. 1996). Personal observations and several local studies have suggested that the long-spined sea urchin Diadema antillarum Philippi has experienced a significant increase in abundance throughout the central-east Atlantic (FAO fisheries region \#34) in the last decades (Casañas et al. 1998, Alves et al. 2001, Tuya et al. 2004b). Although D. antillarum has been extensively studied in the western Atlantic, where it has a great impact on benthic community structure (e.g. Sammarco et al. 1974, Carpenter 1981, 1984, Lessios 1988), little research has been undertaken in the eastern Atlantic to link the demographic structure of this echinoid species with the abundance and biomass of rocky-reef fish assemblages in the Canarian Archipelago. Moreover, no study has addressed the above along with the coastal trophic cascades within this area (Pinnegar et al. 2000).

The general aim of this study was therefore to determine whether there is an association between the structure of Diadema antillarum populations and the community structure of macroalgae and fish. This potential association was examined by quantifying the structure and spatial patterns of $D$. antillarum populations throughout the Canarian Archipelago, and relating this to the fish assemblage structure and macroalgae cover.

\section{MATERIALS AND METHODS}

Experimental design and sampled locations. Since the dynamics of populations involved in trophic interactions operate at different spatial scales (see the review by García-Charton \& Pérez-Ruzafa 1999), we adopted a multiscaled perspective through a hierarchical sampling design with randomly positioned study locations throughout the Canarian Archipelago (Underwood 1997, Kingsford \& Battershill 1998). We selected 4 random locations of rocky substrate in each of the 8 islands of the Archipelago (Fig. 1), with the exception of Chinijo (a group of small islets) and El Hierro Island, where 7 and 5 locations were surveyed, respectively. Furthermore, within each location we randomly sampled 2 sites separated by hundreds of meters in order to increase spatial replication at a small spatial-scale (Kingsford \& Battershill 1998). The locations are referred to numerically (1 to 36 ) and correspond to the geographic locations described in Fig. 1.

All subtidal sampling was conducted from February to May 2003, between 10 and $18 \mathrm{~m}$ depth, along rocky bottoms with similar slope to minimize the effect of habitat type on the distribution and patchiness of assemblages (García-Charton \& Pérez-Ruzafa 1999). Visibility ranged between 8 and $20 \mathrm{~m}$; water temperature between 18 (eastern islands) and $20^{\circ} \mathrm{C}$ (western islands). Unfortunately, no capture and fishing effort data from the artisanal coastal fisheries are available to assign a fishing status to each location (Bas et al. 1995, Pajuelo \& Lorenzo 1995).

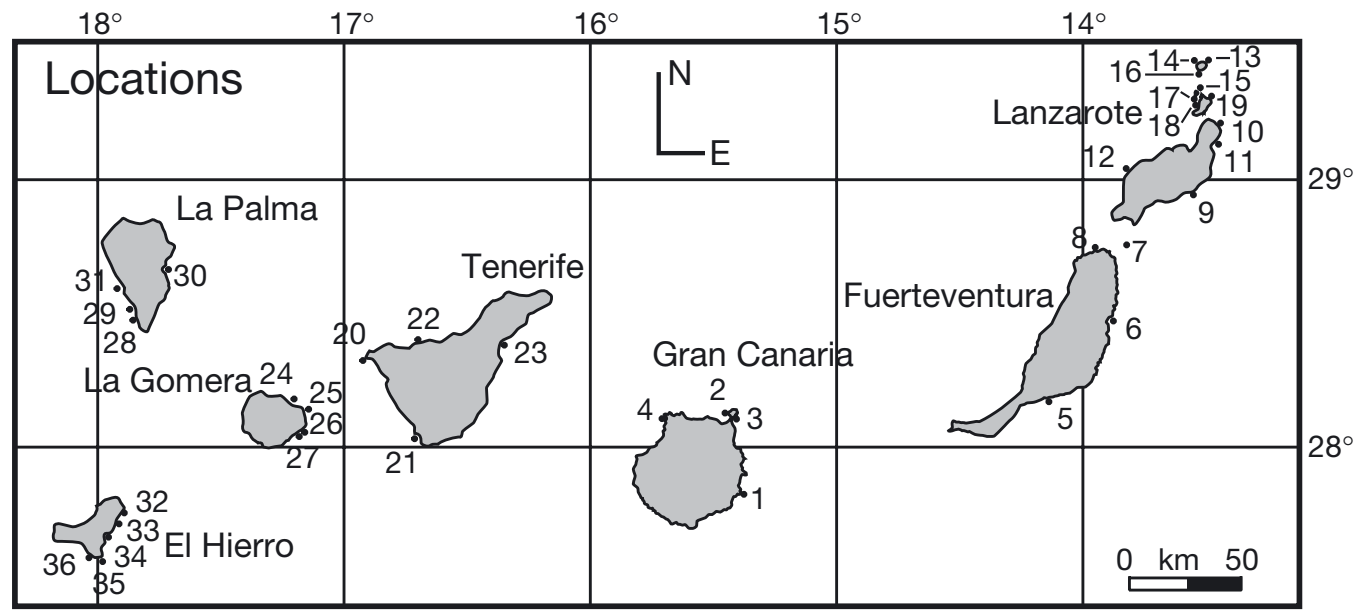

$\begin{array}{cl}\text { Code Location } \\ 1 & \text { El Cabròn } \\ 2 & \text { Canteras } \\ 3 & \text { Puerto deportivo Las Palmas } \\ 4 & \text { Sardina } \\ 5 & \text { Caleta Fuste } \\ 6 & \text { Puerto Rosario } \\ 7 & \text { Lobos } \\ 8 & \text { Corralejo } \\ 9 & \text { Puerto Naos }\end{array}$

$\begin{array}{ll}10 & \text { Orzola } \\ 11 & \text { Arrieta } \\ 12 & \text { Timanfaya } \\ 13 & \text { Miraflores } \\ 14 & \text { Greta } \\ 15 & \text { Roque } \\ 16 & \text { Puerto Viejo } \\ 17 & \text { Veril del Agua } \\ 18 & \text { Ganado }\end{array}$

10 Orzola

12 Timanfaya

13 Miraflores

14 Greta

16 Puerto Viejo

18 Ganado
19 Playa Lambra

20 Teno

21 Christianos

22 Garachico

23 Naútico

24 Hermigua

25 Puntallana

26 Escollera San Sebastián
27 San Sebastián
Fig. 1. The Canarian Archipelago showing sampling locations at each island 
Fish assemblage structure. Non-cryptic fish populations (size $>2 \mathrm{~cm}$ ) were sampled by means of visual census techniques. At each sampling site within each location, 4 replicates of $25 \mathrm{~m}$ long transects were haphazardly laid during daylight hours. The abundance and size (total length to the nearest $2 \mathrm{~cm}$ ) of each fish species within $2 \mathrm{~m}$ of either side of the transects $\left(100 \mathrm{~m}^{2}\right)$ was recorded on waterproof paper by a SCUBA diver, according to standard procedures (Brock 1982, Lincoln-Smith 1988, 1989, Kingsford \& Battershill 1998). Fish belonging to the genera Seriola, Gobius, and Trachurus could not be identified to species visually and were recorded as the genera. Nevertheless, each was then treated as a distinct species in the statistical analyses. Estimation of fish abundance was based on a modification of the method presented by Harmelin-Vivien et al. (1985). Therefore, when fish were grouped in schools larger than 20 individuals, their numbers were estimated according to 6 abundance classes $(20-40,40-70,70-150,150-300$, $300-700,>700$ ).

We calculated the following for each location: (1) the species richness $(S),(2)$ the Shannon-Wiener $\left(H^{\prime}\right)$ diversity index and (3) Pielou's evenness or electivity index $\left(J^{\prime}\right)$ (Ludwig \& Reynolds 1988). Biomass of each species was calculated using the available length-weight relationships for the Canarian Archipelago and from other published and web-based sources (www.fishbase.org). In the cases where length-weight information did not exist for a given species, the parameters from similar bodied congeners were used (Friedlander \& DeMartini 2002). Lengths were first estimated by assigning each fish to the midpoint of its observed size range (Miller \& Gerstner 2002). All measured biotic variables were calculated to a $100 \mathrm{~m}^{2}$ area.

Despite the lack of rigorous knowledge of potential predators of Diadema antillarum along the Canaries, fish species were grouped for statistical procedures into 5 trophic groups (see Table 1) based on previously published diet and feeding habit information (www. fishbase.org), using a similar criterion to that reported for the Atlantic (Jennings et al. 1995) and the Mediterranean (Bell \& Harmelin-Vivien 1983). The 5 groups are: (1) Macroinvertebrate feeders and piscivorous feeders (MaF\&PFs), (2) Macroinvertebrate feeders (MaFs), (3) Microinvertebrate feeders (MiFs), (4) Planktivorous (Ps) and (5) Omnivorous (Os). Therefore, Diadema antillarum can be consumed only by the MaF\&PFs and MaFs groups. Both groups are top predators highly targeted by fishermen throughout the Canarian Archipelago.

Structure of Diadema antillarum populations and macroalgal coverage. The abundance and size class of all Diadema antillarum individuals were visually esti- mated by SCUBA divers using haphazardly located $2 \times 2 \mathrm{~m}\left(4 \mathrm{~m}^{2}\right)$ quadrats in each site $(\mathrm{n}=8)$ within each location (Ruitton et al. 2000). Sea urchins were grouped into 4 size classes (Class $1<1.5 \mathrm{~cm}$ test diameter without spines, Class 2 between 1.5 and $3.5 \mathrm{~cm}$, Class 3 between 3.5 and $5.5 \mathrm{~cm}$ and Class $4>5.5 \mathrm{~cm}$ ) (Casañas et al. 1998). Urchin-grazed barrens were classified for further analysis into 4 categories according to mean density (Casañas et al. 1998) $(<2,2$ to 4 , 4 to 8 and $>8$ ind. $\mathrm{m}^{-2}$ ).

The percentage of fleshy macroalgal cover (mainly frondose brown species belonging to the genera Cystoseira, Sargassum, Lobophora and Dictyota) was visually quantified within each quadrat (Dethier et al. 1993, Benedetti-Cecchi et al. 1996). Final values were expressed as percentages.

Habitat complexity. To assess the effect of the intermediate-to-small spatial-scale substrate complexity on the local dispersion patterns of Diadema antillarum, we counted the number of big $(>1 \mathrm{~m})$ and medium $(0.2$ to $1 \mathrm{~m})$ boulders and crevices in each quadrat, using an approach similar to that reported for the western Mediterranean (García-Charton \& PérezRuzafa 1998) and Madeira Island (Alves et al. 2001). We also obtained the index of rugosity of the substratum through the rope-and-chain technique by using two $1 \mathrm{~m}$ long tapes with a perpendicular distribution within each quadrat (Luckhurst \& Luckhurst 1978, Kingsford \& Battershill 1998).

Statistical analysis. Square-root transformed data of fish abundance was analysed using non-metric multidimensional scaling (nMDS) (Clarke 1993), by means of the PRIMER $^{\odot}$ statistical package, to assess the differences in fish community structure among locations. The 1-way ANOSIM (analysis of similarities) permutation test was used to find the significance of the difference between the locations surveyed within the eastern islands and those along the western islands. The SIMPER procedure was used to identify the contribution of individual species to differences between both groups of islands.

A 3-way nested ANOVA design was used to test for differences in the mean sea urchin density among islands $\left(10^{4}\right.$ to $\left.10^{5} \mathrm{~m}\right)$, locations within islands $\left(10^{3}\right.$ to $\left.10^{4} \mathrm{~m}\right)$ and sites within locations $\left(10^{2} \mathrm{~m}\right)$. Both factors 'locations' and 'sites' were random factors, while 'islands' was considered fixed.

Variance-to-mean ratios $\left(\mathrm{s}^{2} / \mathrm{x}\right.$, index of dispersion, sensu Ludwig \& Reynolds 1988) were calculated to assess the local spatial dispersion pattern (uniform/ clumped/random) of total Diadema antillarum individuals for each location. These values were compared to a 2-tailed $\chi_{n-1}^{2}$ distribution to test for departures from randomness, following a Poisson distribution as a measure of the spatial pattern (Ludwig \& Reynolds 1988). 
Table 1. Mean abundance $\left(\mathrm{n}=8,100 \mathrm{~m}^{2}\right)$ of fish species at the 36 surveyed locations throughout

\begin{tabular}{|c|c|c|c|c|c|c|c|c|c|c|c|c|c|c|c|}
\hline $\begin{array}{l}\text { Trophic group } \\
\text { Species }\end{array}$ & 1 & 2 & 3 & 4 & 5 & 6 & 7 & 8 & 9 & 10 & 11 & 12 & 13 & 14 & 15 \\
\hline \multicolumn{16}{|l|}{ Omnivorous } \\
\hline Sparisoma (Euscarus) cretense & 13.38 & 0.25 & 5.00 & 0.25 & 0.13 & 0.13 & 0.75 & 0.38 & 1.25 & 0.38 & 0.50 & 0.25 & 1.88 & 5.88 & 10.00 \\
\hline Diplodus sargus cadenati & 1.25 & 4.38 & 15.50 & 1.50 & 0.88 & - & 7.88 & - & 0.13 & 1.88 & - & - & - & - & 1.25 \\
\hline Diplodus vulgaris & 0.63 & 1.13 & 6.00 & 0.88 & - & - & 2.75 & - & 0.13 & - & 1.13 & - & - & - & 2.50 \\
\hline Diplodus annularis & 0.25 & - & - & - & - & 0.25 & - & - & - & - & - & - & - & - & - \\
\hline \multicolumn{16}{|l|}{ Microinvertebrate feeders } \\
\hline Thalassoma pavo & 14.63 & 30.25 & 28.50 & 14.00 & 26.38 & 0.13 & 15.00 & 26.38 & 4.25 & 15.63 & 14.00 & 53.63 & 45.00 & 30.88 & 97.13 \\
\hline Abudefduf luridus & 12.25 & 22.50 & 12.50 & 11.88 & 12.38 & 7.38 & 9.25 & 19.88 & 7.50 & 2.50 & 6.00 & 22.75 & 25.00 & 18.75 & 12.75 \\
\hline Canthigaster rostrata & 2.13 & 1.25 & 0.25 & 1.25 & 1.00 & 1.88 & 0.63 & 0.13 & 0.25 & - & 1.50 & 0.50 & - & - & - \\
\hline Scorpaena maderensis & 0.13 & 0.25 & - & 0.13 & 0.25 & - & 0.13 & 0.75 & 0.38 & 0.25 & - & - & 0.63 & - & - \\
\hline Sphoroides spengleri & 2.25 & 0.38 & - & - & - & 0.25 & 0.00 & - & - & - & - & - & - & - & - \\
\hline Apogon imberbis & - & - & - & 0.13 & - & - & - & - & 0.13 & - & - & - & - & - & - \\
\hline Gobius sp. & 0.38 & - & 0.13 & - & - & - & - & - & - & - & - & - & - & - & - \\
\hline \multicolumn{16}{|l|}{ Planktivorous } \\
\hline Boop boops & - & - & 2.00 & - & 462.50 & 1.25 & - & - & 2.50 & - & - & 262.50 & 18.75 & 18.75 & 565.00 \\
\hline Chromis limbatus & 121.25 & - & 112.25 & 43.88 & 100.50 & 6.25 & 145.13 & 32.75 & 37.50 & - & 10.00 & 19.25 & 15.00 & 2.50 & 0.50 \\
\hline Athrerina presbyter & - & - & - & - & - & - & - & - & - & - & - & - & - & - & - \\
\hline \multicolumn{16}{|l|}{ Macroinvertebrate feeders } \\
\hline Coris julis & 18.75 & - & - & 1.75 & - & 0.63 & 1.50 & 0.38 & 7.38 & - & 0.38 & 3.00 & 0.38 & 5.63 & 1.38 \\
\hline Oblada melanura & - & - & 10.00 & 0.13 & - & - & - & - & - & - & 2.50 & - & - & - & - \\
\hline Mullus surmuletus & - & - & - & 0.88 & 0.13 & - & - & - & - & - & 2.50 & - & - & - & - \\
\hline Pomadasys incisus & - & - & - & - & - & - & - & - & 1.00 & - & - & - & - & - & - \\
\hline Aulostomus strigosus & - & - & - & - & - & - & - & - & - & - & - & - & - & - & - \\
\hline Centrolabrus trutta & - & 1.13 & 1.00 & - & - & - & - & - & - & 9.63 & - & - & 0.50 & 1.75 & 1.88 \\
\hline Aluterus sp. & - & - & - & - & - & - & - & - & - & - & 5.00 & - & - & - & - \\
\hline Stephanolepis hispidus & 0.25 & 0.25 & 0.13 & - & - & 2.00 & - & - & - & - & - & - & - & - & - \\
\hline Bothus podas maderensis & - & - & 0.13 & - & - & - & - & - & - & - & - & - & - & - & - \\
\hline Chilomycterus atringa & - & - & - & - & - & - & - & - & - & - & - & - & - & - & - \\
\hline Xyrichthys novacula & - & - & - & - & - & - & - & - & - & - & 0.13 & - & - & - & - \\
\hline Heteropriacanthus cruentatus & - & - & - & - & - & - & - & - & - & - & - & - & - & - & - \\
\hline Lithognathus mormyrus & - & - & - & - & - & - & - & - & - & - & - & - & - & - & - \\
\hline Myrichthys pardalis & - & 0.13 & - & - & - & - & - & - & - & - & - & - & - & - & - \\
\hline \multicolumn{16}{|c|}{ Macroinvertebrate feeders and piscivorous } \\
\hline Sphyraena viridensis & 22.50 & - & - & - & - & - & - & - & - & 1.00 & - & - & - & - & - \\
\hline Pseudocaranx dentex & - & - & 0.75 & - & - & - & - & - & - & - & - & - & - & - & - \\
\hline Serranus atricauda & 0.50 & - & - & - & - & - & - & 0.25 & - & - & - & 0.63 & 0.38 & 0.38 & - \\
\hline Mycteroperca fusca & 0.13 & 0.13 & 0.13 & 0.25 & - & - & - & - & - & - & - & - & - & 0.25 & - \\
\hline Diplodus cervinus & 0.63 & 0.25 & - & 0.50 & 0.13 & - & 1.00 & - & - & - & - & - & 0.63 & - & 0.25 \\
\hline Sarda sarda & - & - & - & - & - & - & - & - & - & - & - & - & - & 9.38 & 0.75 \\
\hline Seriola sp. & - & - & - & - & - & - & - & - & - & - & - & - & - & - & - \\
\hline Kyphosus sectator & - & - & - & - & - & - & - & - & - & - & - & - & - & - & - \\
\hline Spondyliosoma cantharus & - & - & - & - & - & 0.13 & - & - & - & - & - & - & - & - & - \\
\hline Serranus cabrilla & 0.13 & - & - & 0.25 & - & 0.25 & 0.38 & - & - & - & - & - & - & - & - \\
\hline Bodianus scrofa & - & - & - & - & - & - & 0.13 & - & - & - & - & 0.13 & 0.40 & - & - \\
\hline Synodus synodus & - & 0.75 & 0.38 & 0.38 & 0.13 & 0.25 & - & - & - & - & 0.13 & - & - & - & - \\
\hline Serranus scriba & 0.38 & 0.13 & - & 0.13 & - & 0.38 & - & - & - & - & - & - & - & 0.13 & 0.13 \\
\hline Scorpaena porcus & 0.13 & 0.25 & 0.13 & - & - & - & - & 0.50 & - & - & - & - & - & - & - \\
\hline Epinephelus marginatus & - & - & - & - & - & - & - & - & - & - & - & - & 0.13 & - & - \\
\hline Dentex sp. & 0.50 & - & - & - & - & - & - & - & - & - & - & - & - & - & - \\
\hline Dicentrarchus labrax & - & - & 0.50 & - & - & - & - & - & - & - & - & - & - & - & - \\
\hline Gymnothorax unicolor & - & - & - & - & - & - & - & - & - & - & - & - & - & - & - \\
\hline Muraena augusti & - & - & - & - & 0.13 & - & - & - & - & - & - & - & 0.25 & - & - \\
\hline Pagrus auriga & - & - & - & 0.13 & - & - & - & - & - & - & - & - & 0.13 & - & - \\
\hline Synodus saurus & - & - & 0.13 & 0.13 & - & - & - & - & - & - & - & - & - & - & - \\
\hline Muraena helena & 0.13 & - & - & - & - & - & - & - & - & - & - & - & - & - & - \\
\hline Pagrus pagrus & 0.13 & - & - & - & - & - & - & - & - & - & - & - & - & - & - \\
\hline Labrus bergylta & - & - & - & - & - & - & - & - & - & - & - & - & - & - & 0.13 \\
\hline Balistes carolinensis & - & - & - & - & - & - & - & - & - & - & - & - & - & - & - \\
\hline Squatina squatina & - & - & - & - & - & - & - & - & - & - & - & - & 0.13 & - & - \\
\hline Taenuria grabata & - & - & - & - & - & - & - & - & - & - & - & - & - & 0.13 & - \\
\hline Trachinus draco & - & - & - & - & - & - & - & - & - & - & - & - & - & - & - \\
\hline
\end{tabular}


the Canarian Archipelago. Locations numbered as in Fig. 1. Ov. m. ab. = overall mean abundance

\begin{tabular}{|c|c|c|c|c|c|c|c|c|c|c|c|c|c|c|c|c|c|c|c|c|c|}
\hline 16 & 17 & 18 & 19 & 20 & 21 & 22 & 23 & 24 & 25 & 26 & 27 & 28 & 29 & 30 & 31 & 32 & 33 & 34 & 35 & 36 & $\begin{array}{c}\text { Ov. } \\
\text { m.ab. }\end{array}$ \\
\hline 3.00 & 0.75 & 4.88 & 0.13 & 0.88 & 13.38 & 12.13 & 3.63 & 1.88 & 20.63 & 25.38 & 77.38 & 6.25 & 19.00 & 0.88 & 49.38 & 14.38 & 29.63 & 16.63 & 20.75 & 172.75 & 14.833 \\
\hline 1.00 & 2.50 & 0.50 & - & - & 2.63 & 3.13 & 50.38 & - & - & 1.13 & 0.13 & 0.13 & - & 2.25 & 13.75 & - & 0.25 & - & 1.50 & 0.25 & 3.170 \\
\hline 8.88 & 5.38 & - & - & - & 35.50 & 0.13 & 2.63 & - & - & - & 0.63 & - & - & 2.75 & - & - & 2.50 & - & 3.25 & - & 2.132 \\
\hline- & - & - & - & - & - & - & 0.50 & - & - & - & - & - & - & - & - & - & - & - & - & - & 0.028 \\
\hline 31.63 & 14.25 & 16.88 & 12.00 & 102.38 & 18.63 & 13.00 & 44.38 & 45.00 & 67.75 & 13.75 & 79.50 & 40.75 & 52.50 & 11.134 & 466.88 & 536.25 & 396.50 & 47.38 & 97.25 & 286.63 & 78.059 \\
\hline 19.75 & 27.88 & 2.25 & 11.75 & 174.75 & 38.50 & 34.13 & 47.50 & 53.75 & 50.13 & 46.63 & 25.00 & 39.25 & 65.00 & 21.00 & 57.50 & 186.25 & 68.50 & 106.25 & 29.00 & 74.75 & 38.410 \\
\hline 0.50 & 0.38 & 0.13 & 0.25 & 2.88 & 5.63 & 3.25 & 2.50 & 1.50 & 0.13 & 2.38 & 3.13 & 1.75 & 2.00 & 4.38 & 2.25 & 10.25 & 3.25 & 4.38 & 3.00 & 4.75 & 1.927 \\
\hline 0.63 & 0.25 & - & 0.25 & 0.13 & 0.25 & - & 0.13 & - & - & 0.13 & 0.38 & 0.25 & - & 0.25 & 0.38 & 0.50 & - & 0.75 & 0.25 & 0.25 & 0.212 \\
\hline- & - & - & - & 0.38 & - & 0.13 & - & - & - & - & - & 0.13 & - & - & - & - & 0.25 & - & 0.13 & - & 0.108 \\
\hline- & - & - & - & - & - & - & 0.13 & - & - & - & - & - & - & - & - & 1.50 & 0.25 & - & - & - & 0.059 \\
\hline- & - & - & - & - & - & - & - & - & - & - & - & - & - & - & - & - & - & - & - & - & 0.014 \\
\hline- & - & - & 125.00 & 18.75 & - & - & 155.00 & 55.00 & 51.25 & 256.25 & - & - & 32.50 & 325.00 & - & 68.88 & - & 137.50 & - & - & 71.066 \\
\hline 39.38 & 69.00 & 13.88 & 8.63 & 201.25 & 28.50 & - & 76.252 & 201.25 & 130.00 & 35.63 & 0.63 & 263.75 & 276.25 & 17.38 & 4.88 & 1.38 & 24.501 & 100.00 & 20.25 & 12.13 & 60.323 \\
\hline- & - & - & - & - & - & 37.50 & - & - & - & - & - & - & - & 17.50 & - & - & - & 281.25 & - & - & 9.340 \\
\hline 0.13 & 0.63 & 11.00 & - & 9.38 & - & - & - & 3.00 & - & - & - & - & - & - & 1.50 & 2.63 & 0.50 & 5.13 & 1.00 & 4.13 & 2.226 \\
\hline- & 2.50 & - & - & - & - & - & 10.00 & 5.00 & 2.50 & 0.13 & - & 5.00 & 5.00 & 9.00 & - & - & 13.00 & - & 12.88 & - & 2.156 \\
\hline 2.25 & - & - & - & - & 0.13 & - & 56.38 & - & - & 1.50 & - & 0.13 & - & 4.25 & - & - & 3.63 & - & - & - & 1.993 \\
\hline- & - & - & - & 43.75 & - & - & 2.88 & - & - & - & - & - & - & - & - & - & - & - & - & - & 1.323 \\
\hline- & - & - & - & 0.25 & 0.25 & - & - & 0.25 & - & - & 0.25 & 1.13 & 0.63 & - & 4.50 & 4.13 & 6.38 & 2.75 & 12.38 & 1.75 & 0.962 \\
\hline- & - & 4.00 & - & - & - & - & - & - & - & - & - & - & - & - & - & - & - & - & - & - & 0.552 \\
\hline- & - & - & - & - & - & - & - & - & - & - & - & - & - & - & - & - & - & - & - & 0.13 & 0.142 \\
\hline- & - & - & - & - & - & - & - & - & - & - & 0.88 & - & - & - & - & - & - & - & 0.88 & 0.13 & 0.125 \\
\hline- & - & - & - & - & - & - & - & - & - & - & - & - & - & 0.13 & - & - & - & - & - & - & 0.007 \\
\hline- & - & - & - & - & - & - & - & - & - & - & - & - & - & - & - & - & - & 0.25 & - & - & 0.007 \\
\hline- & - & - & - & - & - & - & - & - & 0.13 & - & - & - & - & - & - & - & - & - & - & - & 0.007 \\
\hline- & - & - & - & - & - & - & - & - & - & - & - & - & 0.13 & - & - & - & - & - & - & - & 0.003 \\
\hline- & - & - & - & - & - & - & 0.13 & - & - & - & - & - & - & - & - & - & - & - & - & - & 0.003 \\
\hline- & - & - & - & - & - & - & - & - & - & - & - & - & - & - & - & - & - & - & - & - & 0.003 \\
\hline- & - & - & - & - & - & - & - & - & - & 40.88 & - & - & - & - & - & - & - & - & 4.75 & - & 1.920 \\
\hline- & - & - & - & - & - & - & 18.00 & - & - & 3.25 & 0.25 & - & - & - & - & 0.50 & 6.25 & - & 0.13 & - & 0.809 \\
\hline- & 0.25 & 0.13 & - & 1.13 & 1.50 & - & - & 1.88 & 3.00 & 0.25 & - & 1.13 & 0.38 & - & 1.63 & 1.50 & 0.75 & 0.63 & 0.25 & 1.25 & 0.493 \\
\hline 0.13 & - & 0.25 & - & 0.13 & 0.25 & - & - & - & 0.13 & - & 0.38 & 1.75 & 0.75 & 0.25 & 0.25 & 7.63 & 0.13 & 2.63 & 0.25 & 0.13 & 0.441 \\
\hline 0.13 & - & - & 0.25 & - & 0.13 & 0.75 & - & 1.00 & - & - & 1.75 & 1.38 & 0.50 & - & - & 0.38 & 2.38 & 0.25 & 0.50 & - & 0.354 \\
\hline- & - & - & - & - & - & - & - & - & - & - & - & - & - & - & - & - & - & - & - & - & 0.281 \\
\hline- & - & - & - & - & - & - & 8.75 & - & - & - & 0.25 & - & - & 0.25 & - & - & - & - & - & - & 0.257 \\
\hline- & - & - & - & - & - & - & - & - & - & - & - & - & - & - & - & 4.00 & - & - & 3.00 & - & 0.194 \\
\hline- & - & - & - & 6.00 & - & - & - & - & - & - & - & - & - & - & - & - & - & - & - & - & 0.170 \\
\hline- & - & - & - & - & 0.13 & - & 0.13 & 1.38 & 0.13 & - & - & - & - & - & - & - & - & - & 0.13 & - & 0.080 \\
\hline 0.13 & - & - & 0.25 & - & - & - & - & - & - & - & - & - & - & - & 0.25 & 1.00 & - & 0.13 & 0.25 & 0.13 & 0.077 \\
\hline- & - & - & 0.13 & - & 0.13 & - & - & - & - & - & 0.25 & 0.13 & - & 0.13 & - & - & - & - & - & - & 0.076 \\
\hline 0.38 & - & 0.13 & - & - & 0.75 & - & - & - & - & - & - & - & - & - & - & - & - & - & - & - & 0.069 \\
\hline- & - & - & - & - & - & - & - & - & 0.13 & - & - & - & - & - & - & - & - & - & - & - & 0.031 \\
\hline- & - & - & - & - & - & - & - & - & - & - & - & - & - & - & 0.25 & - & 0.25 & - & 0.13 & - & 0.021 \\
\hline- & - & - & - & - & - & - & - & - & - & - & - & - & - & - & - & - & - & - & - & - & 0.014 \\
\hline- & - & - & - & - & - & - & - & - & - & - & - & - & - & - & - & - & - & - & - & - & 0.014 \\
\hline- & - & - & - & - & - & - & - & - & - & - & - & 0.25 & - & - & 0.13 & 0.13 & - & - & - & - & 0.014 \\
\hline- & - & - & - & - & - & - & - & - & - & - & - & - & - & - & - & - & - & - & - & 0.13 & 0.014 \\
\hline- & - & - & - & - & 0.13 & 0.13 & - & - & - & - & - & - & - & - & - & - & - & - & - & - & 0.014 \\
\hline- & - & - & - & - & - & - & - & - & - & - & - & - & - & 0.25 & - & - & - & - & - & - & 0.014 \\
\hline- & - & - & - & - & - & - & - & - & - & - & - & - & - & - & - & 0.25 & - & - & - & - & 0.010 \\
\hline- & - & - & - & 0.25 & - & - & - & - & - & - & - & - & - & - & - & - & - & - & - & - & 0.010 \\
\hline- & - & 0.13 & - & - & - & - & - & - & - & - & - & - & - & - & - & - & - & - & - & - & 0.007 \\
\hline- & - & - & - & - & - & - & - & - & - & - & - & - & - & - & - & - & 0.13 & - & - & - & 0.003 \\
\hline- & - & - & - & - & - & - & - & - & - & - & - & - & - & - & - & - & - & - & - & - & 0.003 \\
\hline- & - & - & - & - & - & - & - & - & - & - & - & - & - & - & - & - & - & - & - & - & 0.003 \\
\hline- & - & - & - & - & - & - & - & - & - & - & - & - & - & 0.13 & - & - & - & - & - & - & 0.003 \\
\hline
\end{tabular}


One-way ANOVA models on pooled unbalanced data for all surveyed locations were used to test the effects of 4 defined categories of barrens on differences in the mean total abundance and biomass of each considered fish trophic assemblage. Since no transformation rendered homogeneous variances, the level of significance considered was 0.01 instead of 0.05 (Underwood 1981). Furthermore, multiple comparisons were tested using the Tamhane T2 test via the $\mathrm{SPSS}^{\odot}$ software.

To evaluate the spatial relationships between the density of Diadema antillarum sea urchins at each location and (1) their size structure, (2) the fleshy macroalgal cover and (3) the fish species richness, we performed regression models (Underwood 1997). In addition, we carried out correlation analyses between (1) the structure of $D$. antillarum populations and the abundance and biomass of each fish trophic group at each location, and (2) the local dispersion patterns of sea urchin abundance and the measured complexity of the habitat.

\section{RESULTS}

\section{Fish assemblages}

A total of 55 fish species were observed from the 288 visual surveys that we conducted during the sampling period at the 36 sampling locations along the Canary Islands (Table 1). The plankton-feeders Chromis limbatus and Boop boops were the most abundant pelagic schooling species. The wrasse Thalassoma pavo, damselfish Abudefduf luridus and parrotfish Sparisoma cretense were, in decreasing order, the most abundant species of the demersal nearshore fish assemblage (mean density for the entire study $>10$ ind. $100 \mathrm{~m}^{-2}$, Table 1 ). Furthermore, S. cretense, T. pavo and A. luridus were detected at all locations (100\% occurrence frequency), while C. limbatus and Canthigaster rostrata were observed in 91 and $88 \%$ of the locations, respectively. The average number of species per location ( $S$, Table 2) varied between 3.63 (Playa Lambra, Location 19) and 11 at El Rincón (Location 35). Mean species diversity $\left(H^{\prime}\right.$, Table 2$)$ fluctuated between 0.33 (El Roque, Location 15) to 0.72 at Los Cristianos (Location 21),

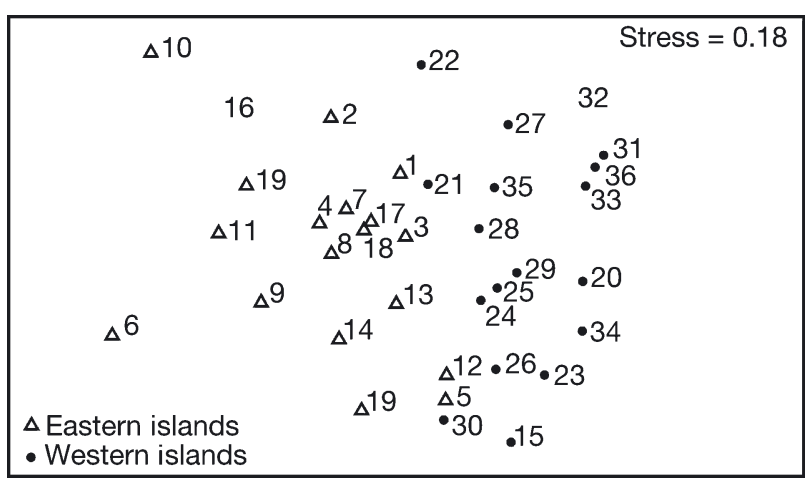

Fig. 2. nMDS plot ordination of the fish species abundance data matrix for all locations sampled

and evenness $\left(J^{\prime}\right.$, Table 2) between 0.40 (Charco Verde, Location 31) and 0.79 (Puerto Rosario and Los Cristianos, Locations 6 and 21). Finally, mean total fish

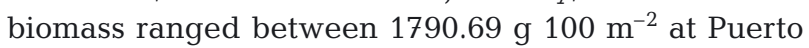

Table 2. Fish descriptive statistics $100 \mathrm{~m}^{-2}$ (diversity indices) and the mean total fish biomass $100 \mathrm{~m}^{-2}$ for each location studied

\begin{tabular}{|c|c|c|c|c|}
\hline Location & $\begin{array}{l}\text { Mean richness } \\
(S)\end{array}$ & Mean $H^{\prime}$ & Mean $J^{\prime}$ & $\begin{array}{l}\text { Mean total biomass } \\
\quad\left(\mathrm{g} 100 \mathrm{~m}^{-2}\right)\end{array}$ \\
\hline 1 & 9.38 & 0.48 & 0.49 & 38791.77 \\
\hline 2 & 7.13 & 0.52 & 0.62 & 8748.67 \\
\hline 3 & 7.75 & 0.60 & 0.68 & 18019.14 \\
\hline 4 & 7.13 & 0.57 & 0.70 & 5948.24 \\
\hline 5 & 5.38 & 0.35 & 0.49 & 54084.67 \\
\hline 6 & 4.88 & 0.52 & 0.79 & 1790.69 \\
\hline 7 & 6.13 & 0.44 & 0.58 & 14615.08 \\
\hline 8 & 3.88 & 0.39 & 0.72 & 5736.43 \\
\hline 9 & 4.88 & 0.42 & 0.61 & 4022.55 \\
\hline 10 & 4.13 & 0.45 & 0.76 & 5213.51 \\
\hline 11 & 4.50 & 0.48 & 0.75 & 6080.94 \\
\hline 12 & 4.88 & 0.34 & 0.54 & 46819.77 \\
\hline 13 & 4.88 & 0.34 & 0.53 & 10343.88 \\
\hline 14 & 4.88 & 0.38 & 0.53 & 28824.78 \\
\hline 15 & 5.75 & 0.33 & 0.43 & 76004.25 \\
\hline 16 & 6.63 & 0.56 & 0.70 & 8556.03 \\
\hline 17 & 4.38 & 0.36 & 0.57 & 8979.03 \\
\hline 18 & 5.75 & 0.52 & 0.70 & 6226.89 \\
\hline 19 & 3.63 & 0.35 & 0.74 & 16174.77 \\
\hline 20 & 4.34 & 0.47 & 0.57 & 61879.26 \\
\hline 21 & 8.50 & 0.72 & 0.79 & 16759.84 \\
\hline 22 & 5.38 & 0.48 & 0.69 & 9200.79 \\
\hline 23 & 8.88 & 0.69 & 0.75 & 47716.55 \\
\hline 24 & 7.38 & 0.55 & 0.63 & 26452.58 \\
\hline 25 & 5.63 & 0.51 & 0.70 & 28852.35 \\
\hline 26 & 7.00 & 0.53 & 0.64 & 63742.73 \\
\hline 27 & 7.13 & 0.52 & 0.63 & 28461.93 \\
\hline 28 & 8.38 & 0.49 & 0.53 & 21910.56 \\
\hline 29 & 6.88 & 0.48 & 0.58 & 24367.52 \\
\hline 30 & 8.50 & 0.44 & 0.49 & 9931.08 \\
\hline 31 & 8.50 & 0.37 & 0.40 & 51682.82 \\
\hline 32 & 10.25 & 0.56 & 0.55 & 37106.93 \\
\hline 33 & 10.13 & 0.52 & 0.51 & 68604.10 \\
\hline 34 & 8.38 & 0.46 & 0.50 & 43931.60 \\
\hline 35 & 11.00 & 0.69 & 0.67 & 62164.81 \\
\hline \multirow[t]{2}{*}{36} & 7.38 & 0.49 & 0.58 & 61269.15 \\
\hline & $6.65 \pm 1.97$ & $0.48 \pm 0.09$ & $0.62 \pm 0.10$ & $28583.77 \pm 22358.48$ \\
\hline
\end{tabular}




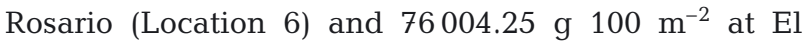
Roque (Location 15), while average total fish biomass

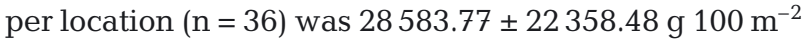
(mean \pm standard deviation) for the entire study.

The 2-dimensional nMDS (Fig. 2) performed on the fish abundances revealed a separation of the locations that lie within the eastern islands (left-side of the plot) from those within the western islands (right-side of the plot). This difference was statistically demonstrated by the 1-way ANOSIM permutation test $(\mathrm{r}=0.32, \mathrm{p}=$ 0.001). Since 2 main groups of islands can therefore be biogeographically differentiated along an east-west gradient through the Canaries, we proceeded to analyze the fish species data from the eastern and western islands separately. As the SIMPER procedure indicated, percentages of contribution to dissimilarities (\%) between both groups of islands were partially due to large differences in the abundances of a few low trophiclevel fish species (Chromis limbatus $-7.20 \%$, Coris julis - $5.36 \%$, Diplodus sargus cadenati $-5.02 \%$, D. vulgaris $-4.78 \%$ and Oblada melanura $-4.65 \%$ ).

\section{Structure of Diadema antillarum populations}

The mean density of Diadema antillarum for the overall study (Table 3 ) was $2.92 \pm 3.75$ ind. $\mathrm{m}^{-2}$ (mean \pm standard deviation, $\mathrm{n}=36$ ), which fluctuated between a minimum of 0 at several locations and a maximum of $13.92 \pm 4.48$ ind. $\mathrm{m}^{-2}$ at Teno (Location 20). As indicated by 3 -way nested ANOVA, the distribution of $D$. antillarum showed statistical differences among locations within islands (Table 4).

Significant non-parametric Spearman correlations were detected between the mean \% frequency of the Size classes 2, 3 and 4 and the local density of Diadema antillarum individuals for all studied locations $\left(\mathrm{R}_{\mathrm{S}}=\right.$ 0.56 for Size class $2, R_{S}=0.51$ for Size class 3 and $\mathrm{R}_{\mathrm{S}}=-0.39$ for Size class 4 ). However, no significant relation was observed for newly settled recruits of $D$. antillarum (Size class 1), as no recruits were recorded throughout the study. The non-linear relations between the mean density of $D$. antillarum at each location and the 3 size classes are graphically illustrated in Fig. 3. Locations with low sea urchin density were therefore dominated by large-sized (Size class 4) individuals, while small-sized sea urchins (Size class 2) were found in high abundances along well-developed urchin-dominated barrens $\left(>8\right.$ ind. $\mathrm{m}^{-2}$ ). Finally, the presence of intermediate-sized individuals (Size class 3) was maximal at intermediate sea urchin density (6 to 8 ind. $\mathrm{m}^{-2}$ ).

Overall, Diadema antillarum exhibited a uniform dispersion pattern at only 2 locations, both at El Hierro Island (Locations 32 and 34, Table 3). On the other hand, D. antillarum showed random dispersion patterns at $75 \%$ of studied locations, which were locations practically devoid of sea urchins $\left(<1\right.$ ind. $\left.\mathrm{m}^{-2}\right)$ or well-developed barrens with a high abundance of individuals (>8 ind. $\mathrm{m}^{-2}$ ) (Table 3 ). The remaining locations $(19 \%)$ with clumped patterns were found at locations with small-to-intermediate density values (1 to 2 ind. $\mathrm{m}^{-2}$ ), except Location 7 (Lobos), where we detected a clumped pattern along a barren with high sea urchin abundance.

Table 3. Diadema antillarum. Mean abundance $\left(\mathrm{n}=16, \mathrm{~m}^{-2}\right)$, standard deviation (SD), index of dispersion (id) and spatial distribution pattern for each studied location. Significant non-parametric correlations $\left(\mathrm{R}_{\mathrm{S}}\right)$ with the measured attributes of the physical complexity of the rocky substrate are also displayed. ${ }^{* *} \mathrm{p}<0.01$

\begin{tabular}{|c|c|c|c|c|c|}
\hline Location & $\begin{array}{c}\text { Mean } \\
\text { density } \\
\left(\text { ind. } \mathrm{m}^{-2} \text { ) }\right.\end{array}$ & $\mathrm{SD}$ & $\begin{array}{c}\text { id } \\
\left(s^{2} / x\right)\end{array}$ & $\begin{array}{c}\text { Spatial } \\
\text { distribution } \\
\text { pattern }\end{array}$ & $\begin{array}{l}\text { Significant } \\
\mathrm{R}_{\mathrm{S}}\end{array}$ \\
\hline 1 & 0.03 & 0.12 & 0.48 & Random & \\
\hline 2 & 0.00 & 0.00 & - & - & \\
\hline 3 & 0.10 & 0.22 & 0.48 & Random & \\
\hline 4 & 5.17 & 1.64 & 0.52 & Random & \\
\hline 5 & 7.61 & 1.82 & 0.44 & Random & \\
\hline 6 & 0.89 & 0.74 & 0.61 & Random & \\
\hline 7 & 8.33 & 5.24 & 3.29 & Patchy & $0.81^{\mathrm{a} * *}$ \\
\hline 8 & 6.19 & 3.20 & 1.65 & Random & \\
\hline 9 & 5.67 & 2.12 & 0.79 & Random & \\
\hline 10 & 0.00 & 0.00 & - & - & \\
\hline 11 & 1.66 & 1.81 & 1.98 & Patchy & $0.91^{\mathrm{c} * *}$ \\
\hline 12 & 10.31 & 3.46 & 1.16 & Random & \\
\hline 13 & 9.25 & 2.86 & 0.89 & Random & \\
\hline 14 & 0.08 & 0.31 & 1.25 & Random & \\
\hline 15 & 0.00 & 0.00 & - & - & \\
\hline 16 & 4.95 & 2.94 & 1.75 & Random & \\
\hline 17 & 4.45 & 2.32 & 1.21 & Random & $0.74^{\mathrm{c} * *}$ \\
\hline 18 & 0.00 & 0.00 & - & - & \\
\hline 19 & 10.41 & 3.49 & 1.17 & Random & \\
\hline 20 & 13.92 & 4.48 & 1.44 & Random & \\
\hline 21 & 1.29 & 2.41 & 4.50 & Patchy & \\
\hline 22 & 0.15 & 0.35 & 0.82 & Random & \\
\hline 23 & 0.01 & 0.09 & 0.56 & Random & \\
\hline 24 & 2.55 & 1.84 & 1.33 & Random & \\
\hline 25 & 2.90 & 1.51 & 0.79 & Random & \\
\hline 26 & 0.28 & 0.37 & 0.49 & Random & \\
\hline 27 & 0.38 & 0.72 & 1.36 & Random & \\
\hline 28 & 2.43 & 3.15 & 4.08 & Patchy & $0.98^{\mathrm{b} * *}$ \\
\hline 29 & 2.89 & 2.79 & 2.69 & Patchy & $0.78^{\mathrm{c} * *}$ \\
\hline 30 & 0.29 & 0.36 & 0.45 & Random & \\
\hline 31 & 0.67 & 0.97 & 1.40 & Random & \\
\hline 32 & 0.91 & 0.47 & 0.24 & Uniform & \\
\hline 33 & 0.23 & 0.43 & 0.80 & Random & \\
\hline 34 & 0.18 & 0.21 & 0.25 & Uniform & \\
\hline 35 & 0.04 & 0.13 & 0.42 & Random & \\
\hline 36 & 0.91 & 1.38 & 2.09 & Patchy & $0.60^{\mathrm{a} * *}$ \\
\hline Total & 2.92 & 3.75 & & & \\
\hline \multicolumn{6}{|c|}{$\begin{array}{l}\text { a Significant correlation with no. of large boulders } \\
\text { bSignificant correlation with no. of large crevices } \\
\text { cSignificant correlation with no. medium crevices }\end{array}$} \\
\hline
\end{tabular}


Table 4. Diadema antillarum. Results of nested ANOVA comparing mean densities among islands, locations and sites. ${ }^{* *} \mathrm{p}<0.01$

\begin{tabular}{|lrrc|}
\hline Source of variation & df & MS & \multicolumn{1}{c|}{$F$} \\
\hline Island & 7 & 197.63 & 0.89 \\
Location (Island) & 24 & 222.01 & $55.92^{* *}$ \\
Site (Location(Island)) & 32 & 3.97 & 1.17 \\
Error & 448 & 3.39 & \\
\hline
\end{tabular}

We found significant positive correlations between the distribution of Diadema antillarum individuals and the physical attributes of the rocky substrate only in those locations where $D$. antillarum exhibited patchy dispersion patterns, with the exception of Location 17 (Veril del Agua) (Table 3). However, no significant correlations were obtained for the rugosity of the substrate or for the depth.

\section{Diadema antillarum versus fleshy macroalgae cover}

Mean densities of total Diadema antillarum individuals and mean fleshy macroalgal coverage (mainly large brown macroalgae) showed an overall negative correlation in the studied locations. A non-linear decrease in the mean percentage of fleshy macroalgal cover with increasing mean density of $D$. antillarum was thus observed (Fig. 4). Hence, high fleshy macro-

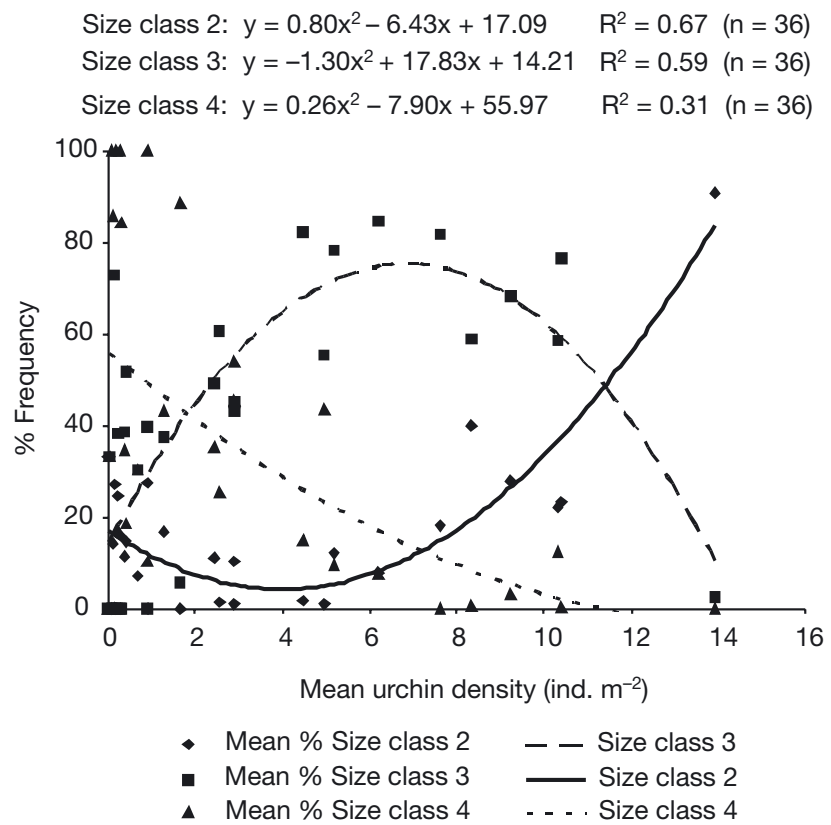

Fig. 3. Diadema antillarum. Relationships between the mean $\%$ frequency of each size class and mean total density of individuals at each location

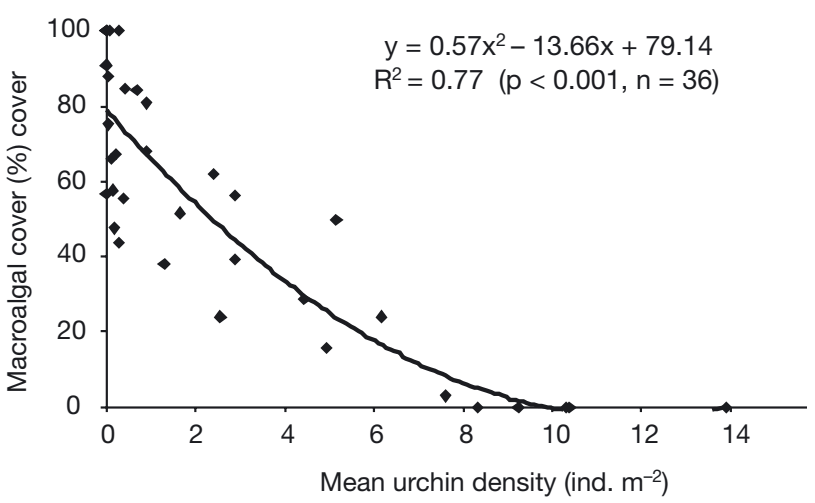

Fig. 4. Diadema antillarum. Non-linear regression between the mean density of total individuals and mean percentage of algal cover at each location

algal cover was found only in those locations where $D$. antillarum was absent or at low densities $\left(<2\right.$ ind. $\left.\mathrm{m}^{-2}\right)$. In contrast, locations with a high density of $D$. antillarum (>8 ind. $\mathrm{m}^{-2}$ ) showed a complete lack of macroalgal cover.

\section{Fish assemblages versus Diadema antillarum density}

Overall, subtidal rocky-bottom fish species richness decreased slightly with an increase in the mean density of Diadema antillarum individuals for both the east and west island groups (Fig. 5).

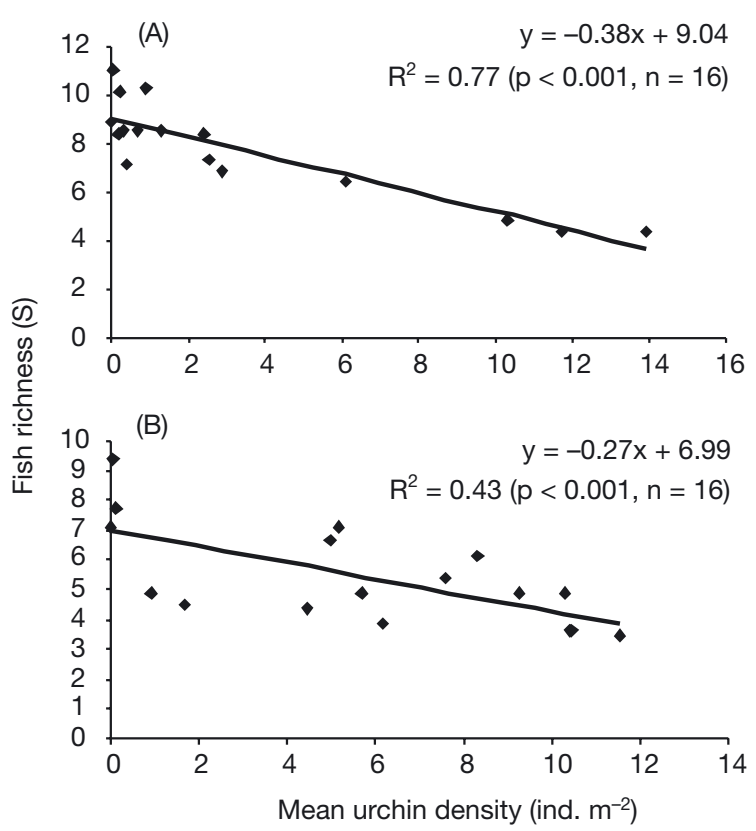

Fig. 5. Diadema antillarum. Relationship between the overall mean density of individuals and rocky-reef fish assemblage richness. (A) Western islands, (B) eastern islands 

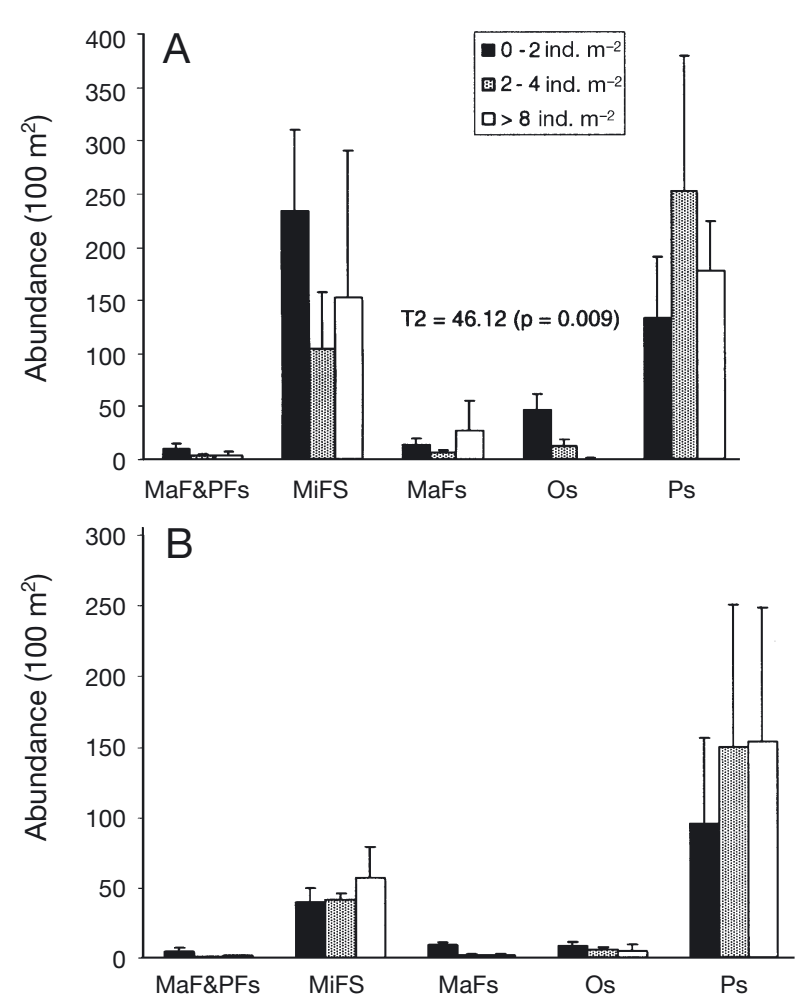

Fig. 6. Total mean abundance of the 5 considered trophic groups with regard to the defined categories of urchin-grazed barrens. Error bars represent SE of means. Above bars: significant results $(\mathrm{p}<0.01)$ of $\mathrm{T} 2$ Tamhane a posteriori multiple comparisons. (A) Western islands, (B) eastern islands. MaF\&PFs: macroinvertebrate feeders and piscivorous, MaFs: macroinvertebrate feeders, MiFs: microinvertebrate feeders, Ps: planktivorous, Os: omnivorous

Differences in the trophic structure of the fish assemblages were observed between the eastern and western islands with regard to the 4 barren categories (Figs. 6 \& 7). However, significant differences were detected by 1 -way ANOVA ( $p<0.01$, Figs. 6 \& 7) only in the mean total abundance and biomass of omnivorous fish species from the western islands, as a consequence of the large and heterogeneous variances associated with the values of mean total abundance and biomass. Nonetheless, the mean biomass of MaF\&PFs is about 9 and 10 times greater in lowurchin density rocky environments $\left(<2\right.$ ind. $\left.\mathrm{m}^{-2}\right)$ than in well developed urchin-grazed barrens ( $>8$ ind. $\mathrm{m}^{-2}$ ) for the eastern and western Canary Islands (Fig. 7), respectively. Likewise, the mean biomass of omnivorous fish species (mainly the parrotfish Sparisoma cretense) for the western and eastern islands was 6 and 2 times greater, respectively, in barrens with low densities of sea urchins that in heavilygrazed barrens. In contrast, the mean biomass of fastgrowing plankton-feeding species (e.g. Chromis limbatus, Atherina presbyter) in overgrazed barrens
(>8 ind. $\mathrm{m}^{-2}$ ) is approximately twice that observed in locations either devoid of or with very low density of Diadema antillarum sea urchins for the eastern islands, and 3 times greater for the western islands (Fig. 7).

The above-mentioned results are corroborated by the significant non-parametric Spearman correlation coefficients found between the overall mean densities of Diadema antillarum individuals and the mean total abundance and biomass of the 5 considered trophic groups (Table 5). Significant negative correlations were observed for both macroinvertebrate-feeder groups (MaF\&PFs and MaFs), as well as for the omnivorous fish group. In addition, the significant correlations between mean abundance and biomass of the 5 considered trophic groups and the 4 defined size classes of D. antillarum (Table 6) seem to indicate an inverse relationship between the mean abundance and biomass of macroinvertebrate-feeders registered per location and the mean \% frequency of the small-tointermediate size classes. On the other hand, we recorded several significant positive correlations between sea urchin density and total mean abundance and biomass of plankton-feeders (Table 5).
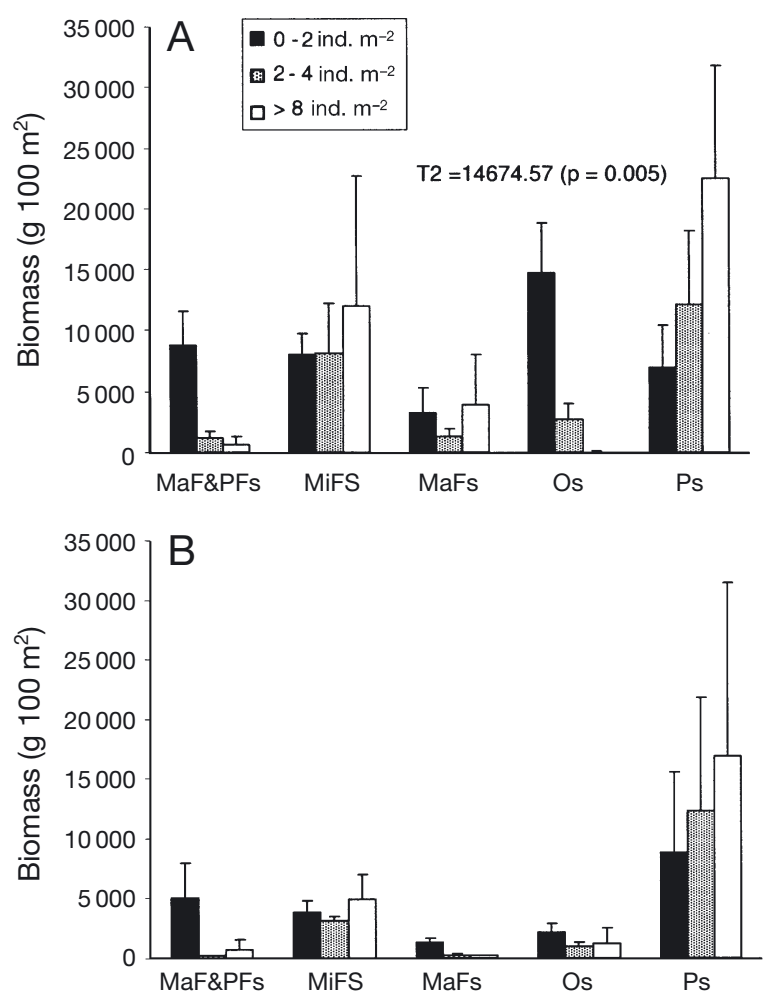

Fig. 7. Total mean biomass of the 5 considered trophic groups with regard to the defined categories of urchin-grazed barrens. Error bars represent SE of means. Above bars: significant results $(\mathrm{p}<0.01)$ of T2 Tamhane a posteriori multiple comparisons. (A) Western islands, (B) eastern islands. MaF\&PFs: macroinvertebrate feeders and piscivorous, MaFs: macroinvertebrate feeders, MiFs: microinvertebrate feeders, Ps: planktivorous, Os: omnivorous 
Table 5. Diadema antillarum. Significant non-parametric Spearman correlation coefficients and levels of significance (in parentheses) of correlations between the mean density of individuals per location and the mean total abundance and biomass of the 5 defined fish trophic groups. Dashed lines indicate no significant relationship

\begin{tabular}{|c|c|c|c|c|c|c|}
\hline & \multicolumn{2}{|c|}{ Overall Archipelago $(\mathrm{n}=36)$} & \multicolumn{2}{|c|}{ Eastern islands $(\mathrm{n}=19)$} & \multicolumn{2}{|c|}{ Western islands $(\mathrm{n}=17)$} \\
\hline & $\begin{array}{l}\text { Mean total } \\
\text { abundance }\end{array}$ & $\begin{array}{l}\text { Mean total } \\
\text { biomass }\end{array}$ & $\begin{array}{l}\text { Mean total } \\
\text { abundance }\end{array}$ & $\begin{array}{l}\text { Mean total } \\
\text { biomass }\end{array}$ & $\begin{array}{l}\text { Mean total } \\
\text { abundance }\end{array}$ & $\begin{array}{c}\text { Mean total } \\
\text { biomass }\end{array}$ \\
\hline MaF\&PFs & $-0.31(0.060)$ & $-0.44(0.006)$ & - & $-0.46(0.047)$ & - & - \\
\hline MiFs & - & - & - & - & - & - \\
\hline MaFs & $-0.41(0.013)$ & $-0.30(0.067)$ & $-0.65(0.002)$ & $-0.58(0.009)$ & - & - \\
\hline Os & $-0.48(0.003)$ & $-0.52(0.001)$ & $-0.55(0.013)$ & $-0.58(0.009)$ & - & $-0.45(0.065)$ \\
\hline Ps & - & $0.34(0.042)$ & $0.45(0.052)$ & $0.45(0.050)$ & - & - \\
\hline
\end{tabular}

Table 6. Diadema antillarum. Significant non-parametric Spearman correlation coefficients and levels of significance (in parentheses) of correlations between the mean \% of frequency of the 4 size classes and the mean total abundance and biomass of the 5 defined fish trophic groups. Dashed lines indicate no significant relationship

\begin{tabular}{|c|c|c|c|c|c|c|c|c|c|c|}
\hline & \multicolumn{5}{|c|}{ Mean total abundance } & \multicolumn{5}{|c|}{ Mean total biomass } \\
\hline & MaF\&PFs & MiFs & MaFs & Os & Ps & MaF\&PFs & MiFs & MaFs & Os & Ps \\
\hline \multicolumn{11}{|c|}{ Overall Canarian Archipelago $(\mathrm{n}=36)$} \\
\hline Size class 1 & - & - & _- & - & _- & _- & - & - & _- & - \\
\hline Size class 2 & - & - & - & - & - & - & - & - & - & - \\
\hline Size class 3 & - & - & $-0.60(0.000)$ & - & - & $-0.48(0.003)$ & - & $-0.54(0.003)$ & - & - \\
\hline Size class 4 & - & - & - & - & - & - & - & - & - & - \\
\hline \multicolumn{11}{|c|}{ Western islands $(\mathrm{n}=17)$} \\
\hline Size class 1 & - & - & - & - & - & - & - & - & - & - \\
\hline Size class 2 & - & - & - & - & - & - & - & $-0.49(0.041)$ & - & - \\
\hline Size class 3 & - & - & - & - & - & $-0.45(0.067)$ & - & - & - & - \\
\hline Size class 4 & - & - & - & - & - & - & - & - & - & - \\
\hline \multicolumn{11}{|c|}{ Eastern islands $(\mathrm{n}=19)$} \\
\hline Size class 1 & - & _- & _- & - & - & - & - & - & - & - \\
\hline Size class 2 & - & - & $-0.64(0.008)$ & - & $0.59(0.015)$ & - & - & $-0.58(0.017)$ & - & - \\
\hline Size class 3 & - & - & $-0.71(0.002)$ & - & - & $-0.57(0.020)$ & - & $-0.64(0.007)$ & - & - \\
\hline Size class 4 & - & - & - & - & - & - & - & - & - & - \\
\hline
\end{tabular}

\section{DISCUSSION}

Our study represents another case in which low abundance and biomass of top predatory fish seem to be related to high densities of sea urchins and in turn, to low cover of fleshy macroalgae. These results suggest that a trophic-cascade may exist in the Canarian Archipelago, as has been demonstrated in other locations worldwide such as the Mediterranean (Sala \& Zabala 1996, Sala et al. 1998), tropical coral-reef habitats (Hay 1984, McClanahan \& Muthiga 1988, McClanahan \& Shafir 1990) and kelp-dominated areas (Andrew \& Choat 1982, Bernstein et al. 1983, Steneck 1997, Babcock et al. 1999, Shears \& Babcock 2003). We suggest that this cascade is at least partially related to overfishing of large macroinvertebrate-eating fish (Duggins 1980, Tegner \& Dayton 1981, Breen et al. 1982, Tegner \& Levin 1983, Hay 1984, McClanahan \& Muthiga 1988, McClanahan \& Shafir 1990, McClanahan 1992, McClanahan et al. 1994, Sala \& Zabala 1996, Babcock et al. 1999); although no data on fishing intensity in this area is available to support this assumption, as artisanal fishermen sell their captures directly to local markets without any sort of governmental con- trol (Bas et al. 1995, Pajuelo \& Lorenzo 1995). The increased prevalence of urchin-dominated barrens throughout the Canarian Archipelago could be therefore considered as one symptom of long-standing intense use (e.g. overfishing) of the littoral (Sala \& Zabala 1996, Sala et al. 1998, Babcock et al. 1999, Pinnegar et al. 2000, Shears \& Babcock 2003).

Our results suggest, therefore, that macroinvertebrate-eating carnivorous fish (e.g. Sparids and Labrids) act as a controlling force on the Diadema antillarum population structure through predation. These species have been widely reported as potential predators of sea urchins (e.g. McClanahan 1995, Sala \& Zabala 1996, Sala 1997). The clear, negative relationship between sea urchin density and the mean total abundance (or biomass) of the omnivorous fish group is probably caused by the most abundant omnivorous species (the parrotfish Sparisoma cretense) inhabiting, almost exclusively, shallow algal bands throughout the Canarian Archipelago (Bortone et al. 1991, Falcón et al. 1996). Additionally, compensatory increases (sensu Myers \& Worm 2003) in fast-growing plankton-feeding coastal fish species (e.g. Chromis limbatus) were observed in our study throughout well- 
developed urchin-dominated barrens of the rocky littoral zones of the Canary Islands.

The high variability in sea urchin density that could not be explained by fish density suggests that other mechanisms also control it. The interaction between sea urchins and coastal fish populations is thus a complex interplay between physical and physiological disturbance, competition, predation and recruitment, which in turn are influenced by stochastic factors (Sala \& Zabala 1996). Hence, factors other than predation-based cascades could contribute to the interaction between Diadema antillarum and hard-bottom fish populations in the first place and, therefore, to the large variability observed in sea urchin density among locations within islands. Processes including pollution, diseases, large-scale oceanographic events, recruitment and the structural complexity of the rocky substratum (availability of refuges) are also important factors driving the dynamic interaction processes between rocky-bottom fish assemblages and sea urchin populations (Sala et al. 1998).

The size structure of Diadema antillarum is dominated by small-to-intermediate sized sea urchins (Size classes 2 and 3) within environments with a high density of individuals. This strategy may buffer the adverse effects of increases in population density and allow exploitation of resources (Levitan 1988, 1991, Karlson \& Levitan 1990, Alves et al. 2001). In contrast, low sea urchin density locations are characterized by the dominance of large sized individuals. The lack of potential predators for large sized $D$. antillarum individuals (Size class 4) could explain this fact. This result contrasts with the size-frequency distributions observed by Sala \& Zabala (1996) for the edible sea urchin Paracentrotus lividus (Lamarck) in the western Mediterranean, where low numbers of large sized sea urchins were recorded within protected areas with high fish density. This is attributable to differences in morphology and therefore to the higher structural complexity of a long-spined sea urchin such as D. antillarum versus a short-spined such as P. lividus. Predation pressure on $D$. antillarum should be thus concentrated on small-to-intermediate sized individuals (Size classes 1 and 2). Consequently, we propose the existence of a predator 'escape size' (sensu Sala 1997) for the sea urchin D. antillarum along the Canarian Archipelago. On the other hand, our correlation-based study has not provided evidence of the existence of different predator groups for differently sized sea urchins, as Sala (1997) has reported for P. lividus. Hence, further research should focus on this point by means of direct observations, predation experiments and gut content analyses (McClanahan \& Muthiga 1988, McClanahan 1995, Sala \& Zabala 1996).

Garrido et al. (2000) have shown that Diadema antillarum presents a reproductive peak in the Canarian coasts during the spring months (April to May); this fact may explain the absence of new recruits (Size class 1) in all locations, as we made our observations in late winter.

The physical complexity of subtidal rocky bottoms, at the small-to-intermediate spatial scale measured by our study, seems to play an important role in determining the local patchiness of Diadema antillarum populations, when density is intermediate. In contrast, habitat complexity is not important throughout welldeveloped urchin-grazed barrens (>8 ind. $\mathrm{m}^{-2}$ ), where $D$. antillarum is randomly distributed and widespread along rocky reefs. We believe that the local complexity of the rocky substrate is therefore important when the populations of $D$. antillarum are under high predator control and, consequently, seek shelter within refuges (e.g. medium and large crevices, inside groups of boulders) during daylight hours to reduce the risk of predation. This crevice-dwelling behavior has been reported in the Caribbean (Carpenter 1981, 1984) and the eastern Atlantic (Tuya et al. 2004a), and is common in other echinoid species in the presence of predators in temperate waters (Andrew \& Underwood 1989, Sala \& Zabala 1996). Hence, the availability of refuges may be a sufficient condition for the creation of areas of barren habitat (Andrew 1993, Sala \& Zabala 1996), even in locations with intense predatory activity such as Location 7 of our study.

The strong negative relationship observed between the percentage of fleshy macroalgal cover (frondose brown algae) and the density of Diadema antillarum has also been described in Caribbean waters (Carpenter 1981, Sammarco 1982, Hay 1984, Lessios et al. 2001) and the eastern Atlantic (Alves et al. 2001, Tuya et al. 2004b); as well as for other echinoid species in the western Mediterranean (Sala \& Boudouresque 1997), the Kenyan coast (McClanahan et al. 1996), New Zealand (Andrew \& Choat 1982, Babcock et al. 1999, Shears \& Babcock 2003) and in the north-western Atlantic (Vadas \& Steneck 1995). The non-linear decrease in fleshy macroalgal cover with increasing sea urchin density has been reported as the response of algae to the in situ manipulation of sea urchin density (Andrew \& Underwood 1993, Benedetti-Cecchi et al. 1998). Although the reasons underlying non-linearities are difficult to interpret (Andrew \& Underwood 1993, Benedetti-Cecchi et al. 1998), we believe that differences in the quality of algae as food resources for sea urchins could explain this process (Benedetti-Cecchi et al. 1998), since sea urchins are able to detect preferred species (Himmelman \& Nédélec 1990). D. antillarum displayed clear feeding preferences over the most abundant large brown macroalgae species of the Canary Islands (Tuya et al. 2001). Therefore, individuals can forage only on food resources of high quality if 
density is low. However, D. antillarum has to graze over a wide variety of algal species when density increases, consuming all available algae and leading to a dramatic decrease in macroalgal cover. Although grazing on macroalgae may change with depth (Ruitton et al. 2000), we can consider the long-spined sea urchin $D$. antillarum as a key herbivorous species (Tuya et al. 2004b) that plays an important role in determining fleshy macroalgae cover in rocky subtidal communities of the Canarian Archipelago.

Acknowledgements. Research was economically supported by the Spanish Ministerio de Medio Ambiente in the framework of the Canarias, por una costa viva project (www.canariasporunacostaviva.org) in collaboration with WWF/Adena. We gratefully thank E. Falcón, O. Bergasa, T. Sánchez, A. Iglesias, A. López, N. Rodriguez, A. Del Rosario, G. Herrera, R. Herrera and F. Espino for helping us with the underwater data collection and processing. Corrections by Dr. T. R. McClanahan and 3 anonymous reviewers significantly improved the paper.

\section{LITERATURE CITED}

Alves FMA, Chicharo LM, Serrao E, Abreu AD (2001) Algal cover and sea urchin spatial distribution at Madeira Island (NE Atlantic). Sci Mar 65:383-392

Andrew NL (1993) Spatial heterogeneity, sea urchin grazing, and habitat structure on reefs in temperate Australia. Ecology 74:292-302

Andrew NL, Choat JH (1982) The influence of predation and conspecific adults on the survivorship of juvenile Evechinus chloroticus (Echinoidea: Echinometridae). Oecologia 54:80-87

Andrew NL, Underwood AJ (1989) Patterns of abundance of the sea urchin Centrostephanus rodgersii (Agassiz) on the central coast of New South Wales, Australia. J Exp Mar Biol Ecol 131:61-80

Andrew NL, Underwood AJ (1993) Density dependent foraging in the sea urchin Centrostephanus rodgersii on shallow subtidal reefs in New South Wales, Australia. Mar Ecol Prog Ser 99:89-98

Babcock RC, Kelly S, Shears NT, Walker JW, Willis TJ (1999) Changes in community structure in temperate marine reserves. Mar Ecol Prog Ser 189:125-134

Bas C, Castro JJ, Hernández-García V, Lorenzo JM, Moreno T, Pajuelo JG, González Ramos AJ (1995) La Pesca en Canarias y áreas de influencia. Ediciones del Cabildo Insular de Gran Canaria, Las Palmas

Bell JD, Harmelin-Vivien ML (1983) Fish fauna of French Mediterranean Posidonia oceanica seagrass meadows. Tethys 11:1-4

Benedetti-Cecchi L, Airoldi L, Abbiati M, Cinelli F (1996) Estimating the abundance of benthic invertebrates: a comparison of procedures and variability between observers. Mar Ecol Prog Ser 138:93-101

Benedetti-Cecchi L, Bulleri F, Cinelli F (1998) Density dependent foraging of sea urchins in shallow subtidal reefs on the west coast of Italy (western Mediterranean). Mar Ecol Prog Ser 163:203-211

Bernstein BB, Schroeter SC, Mann KH (1983) Sea urchin (Strongylocentrotus droebachiensis) aggregating behav- iour investigated by a subtidal multifactorial experiment. Can J Fish Aquat Sci 40:1975-1986

Bortone SA, Van Tasell J, Brito A, Falcón JM, Bundrick CM (1991) A visual assessment of the inshore fishes and fishery resources off El Hierro, Canary Islands: a baseline survey. Sci Mar 55:529-541

Breen PA, Carson TA, Foster JB, Stewart EA (1982) Changes in subtidal community structure associated with British Columbia sea otter transplants. Mar Ecol Prog Ser 7:13-20

Brock RE (1982) A critique of the visual census method for assessing coral reef fish populations. Bull Mar Sci 32: 269-276

Carpenter RC (1981) Grazing by Diadema antillarum and its effects on the benthic algal community. J Mar Res 39: 749-765

Carpenter RC (1984) Predator and population density control of homing behaviour in the Caribbean echinoid Diadema antillarum. Mar Biol 82:101-108

Casañas A, Hanek-Larsen H, Haroun RJ (1998) Developmental stages of blanquizal due to herbivory by the sea urchin Diadema antillarum Philippi in the Canary Islands. Bol Mus Mun Fun 5:139-146

Clarke KR (1993) Non-parametric multivariate analyses of changes in community structure. Aus J Ecol 18:117-143

Dethier MN, Grahan ES, Cohen S, Tear LM (1993) Visual versus random-point percent cover estimations: 'objective' is not always better. Mar Ecol Prog Ser 96:93-100

Duggins DO (1980) Kelp beds and sea otters: an experimental approach. Ecology 61:447-453

Falcón JM, Bortone SA, Brito A, Bundrick CM (1996) Structure and relationships within and between the littoral rock-substrate fish communities off four islands in the Canarian Archipelago. Mar Biol 125:215-231

Francour P (1994) Pluriannual analysis of the reserve effect on fish community in the Scandola natural reserve (Corsica, north-western Mediterranean). Oceanol Acta 17:309-317

Friedlander AM, DeMartini EE (2002) Contrast in density, size, and biomass of reef fishes between the northwestern and the main Hawaiian Islands: the effects of fishing down apex predators. Mar Ecol Prog Ser 230:253-264

García-Charton JA, Pérez-Ruzafa A (1998) Correlation between habitat structure and a rocky reef fish assemblages in SW Mediterranean. PSZN I: Mar Ecol 19:111-128

García-Charton JA, Pérez-Ruzafa A (1999) Ecological heterogeneity and the evaluation of the effects of marine reserves. Fish Res 42:1-20

Garrido M, Haroun RJ, Lessios HA (2000) Annual reproductive periodicity of the sea urchin Diadema antillarum Phillipi in the Canary Islands. Bull Mar Sci 67:989-996

Harmelin-Vivien ML, Harmelin-Vivien JG, Chauvet C, Duval $\mathrm{C}$ and 7 others (1985) Evaluation visuelle des peuplements et populations de poissons: méthodes et problèmes. Terre Vie 40:467-539

Hay ME (1984) Patterns of fish and urchin grazing on Caribbean coral reefs: are previous results typical? Ecology 65:446-454

Himmelman JH, Nédélec H (1990) Urchin foraging and algal survival strategies in intensely grazed communities in eastern Canada. Can J Fish Aquat Sci 47:1011-1026

Jackson JBC (2001) What was natural in the coastal oceans? PNAS 98:5411-5418

Jennings S, Grandcourt EM, Polunin NVC (1995) The effects of fishing on the diversity, biomass and trophic structure of Seychelles' reef fish communities. Coral Reefs 14:225-235

Karlson RH, Levitan DR (1990) Recruitment-limitation in open populations of Diadema antillarum: an evaluation. Oecologia 82:40-44 
Kingsford M, Battershill C (1998) Studying marine temperate environments: a handbook for ecologists. Canterbury University Press, Christchurch, New Zealand

Lessios HA (1988) Population dynamics of Diadema antillarum Philippi (Echinodermata: Echinoidea) following mass mortality in Panama. Mar Biol 99:515-526

Lessios HA, Garrido MJ, Kessing BD (2001) Demographic history of Diadema antillarum, a keystone herbivore on Caribbean reefs. Proc R Soc Lond B 268:1-7

Levitan DR (1988) Density-dependent size regulation and negative growth in the sea urchin Diadema antillarum. Oecologia 76:627-629

Levitan DR (1991) Skeletal changes in the test and jaws of the sea urchin Diadema antillarum in response to food limitation. Mar Biol 111:431-435

Lincoln-Smith MP (1988) Effects of observer swimming speed on sample counts of temperate rocky reef fish assemblages. Mar Ecol Prog Ser 43:223-231

Lincoln-Smith MP (1989) Improving multispecies rocky reef fish censuses by counting different groups of species using different procedures. Environ Biol Fish 26:29-37

Luckhurst BE, Luckhurst K (1978) Analysis of the influence of substrate variables on coral reef fish communities. Mar Biol 49:317-323

Ludwig JA, Reynolds JF (1988) Statistical ecology. John Wiley \& Sons, New York

Mann KH (1982) Kelp, sea urchins and predators: a review of strong interactions in rocky subtidal systems of eastern Canada, 1970-1980. Neth J Sea Res 16:414-423

McClanahan TR (1992) Resource utilization competition and predation: a model and example from coral reef grazers. Ecol Model 61:195-215

McClanahan TR (1995) Fish predators and scavengers of the sea urchin Echinometra mathaei in Kenyan coral-reef marine parks. Environ Biol Fish 43:187-193

McClanahan TR, Kurtis JD (1991) Population regulation of the rocky-boring sea urchin Echinometra mathaei (de Blainville). J Exp Mar Biol Ecol 147:121-146

McClanahan TR, Muthiga NA (1988) Changes in Kenyan coral reefs. Community structure and function due to exploitation. Hydrobiologia 166:269-276

McClanahan TR, Sala E (1997) A Mediterranean rocky-bottom ecosystem fisheries model. Ecol Model 104:145-165

McClanahan TR, Shafir SH (1990) Causes and consequences of sea urchin abundance and diversity in Kenyan coral reefs. Oecologia 83:362-370

McClanahan TR, Nugues M, Mwachireya S (1994) Fish and sea urchin herbivory and competition in Kenyan coral reef lagoons: the role of reef management. J Exp Mar Biol Ecol 184:237-254

McClanahan TR, Kamukuru AT, Muthiga NA, Gilagabher M, Obura D (1996) Effect of sea urchin reductions on algae, coral and fish populations. Conserv Biol 10:136-154

Miller MW, Gerstner CL (2002) Reefs of an uninhabited Caribbean island: fishes, benthic habitat, and opportunities to discern reef fishery impact. Biol Conserv 106:37-44

Myers RA, Worm B (2003) Rapid worldwide depletion of predatory fish communities. Nature 423:280-283

Pajuelo JG, Lorenzo JM (1995) Análisis y predicción de la pesquería demersal de las Islas Canarias mediante un modelo ARIMA. Sci Mar 59:155-164

Pinnegar JK, Polunin NVC, Francour P, Badalamenti F and 7 others (2000) Trophic cascades in benthic marine ecosystems: lessons for fisheries and protected-area management. Environ Conserv 27:179-200

Ruitton S, Francour P, Boudouresque CF (2000) Relationships between algae, benthic herbivorous invertebrates and fishes in rocky sublittoral communities of a temperate sea (Mediterranean). Estuar Coast Shelf Sci 50:217-230

Sala E (1997) Fish predators and scavengers of the sea urchin Paracentrotus lividus in protected areas of the northwestern Mediterranean Sea. Mar Biol 129:531-539

Sala E, Boudouresque CF (1997) The role of fishes in the organization of a Mediterranean sublittoral community. I. Algal communities. J Exp Mar Biol Ecol 212:25-44

Sala E, Zabala M (1996) Fish predation and the structure of the sea urchin Paracentrotus lividus population in the NW Mediterranean. Mar Ecol Prog Ser 140:71-81

Sala E, Boudouresque CF, Harmelin-Vivien ML (1998) Fishing, trophic cascades, and the structure of algal assemblages: evaluation of an old but untested paradigm. Oikos 83:425-439

Sammarco PW (1982) Effects of grazing by Diadema antillarum Philippi (Echinodermata) on algal diversity and community structure. J Exp Mar Biol Ecol 65:83-105

Sammarco PW, Levinton JS, Ogden JC (1974) Grazing and control of coral reef community structure by Diadema antillarum Philippi (Echinodermata: Echinoidea). A preliminary study. J Mar Res 32:47-53

Shears NT, Babcock RC (2003) Continuing trophic cascade effects after 25 years of no-take marine reserve protection. Mar Ecol Prog Ser 246:1-16

Steneck RS (1997) Crustose corallines, other algal functional groups, herbivores and sediment: complex interactions along reef productivity gradients. Proc 8th Int Coral Reef Symp 1:695-700

Tegner MJ, Dayton PK (1981) Population structure, recruitment and mortality of two sea urchins (Strongylocentrotus droebachiensis and S. purpuratus) in kelp forest. Mar Ecol Prog Ser 5:255-268

Tegner MJ, Levin LA (1983) Spiny lobsters and sea urchins: analysis of a predator-prey interaction. J Exp Mar Biol Ecol 73:125-150

Tuya F, Martín JA, Reuss GM, Luque A (2001) Feeding preferences of the sea urchin Diadema antillarum in Gran Canaria Island (Central-East Atlantic Ocean). J Mar Biol Assoc UK 81:1-5

Tuya F, Martín, JA, Luque A (2004a) Patterns of nocturnal movement of the long-spined sea urchin Diadema antillarum (Philippi) in Gran Canaria (the Canary Islands, central East Atlantic Ocean). Helgol Mar Res 58:26-31

Tuya F, Boyra A, Sanchez-Jerez P, Barbera C, Haroun RJ (2004b) Can one species determine the structure of the benthic community on a temperate rocky reef? The case of the long-spined sea urchin Diadema antillarum (Echinodermata: Echinoidea) in the eastern Atlantic. Hydrobiologia 519:211-214

Underwood AJ (1981) Techniques of analysis of variance in experimental marine biology and ecology. Oceanogr Mar Biol Annu Rev 19:513-605

Underwood AJ (1997) Experiments in ecology: their logical design and interpretation using analysis of variance. Cambridge University Press, Cambridge

Vadas RL, Steneck RS (1995) Overfishing and interferences in kelp-sea urchin interactions. In: Hopkins HR, Erikstad C, Leinaas HR (eds) Ecology of fjords and coastal waters. Elsevier, Amsterdam, p 509-524

Verlaque M (1987) Relations entre Paracentrotus lividus (Lamarck) et le phytobenthos de Méditerranée occidentale. In: Boudouresque CF (ed) Colloque international sur Paracentrotus lividus et les oursins comestibles. GIS Posidonie, Marseille, p 5-36

Vukovic A (1982) Florofaunistic changes in the infralittoral zone after the sea urchin Paracentrotus lividus (L.) population explosion. Acta Adriat 23:237-241 